\title{
Geolocalización en las campañas SEM: una mirada al uso de los términos de ubicación en las palabras clave
}

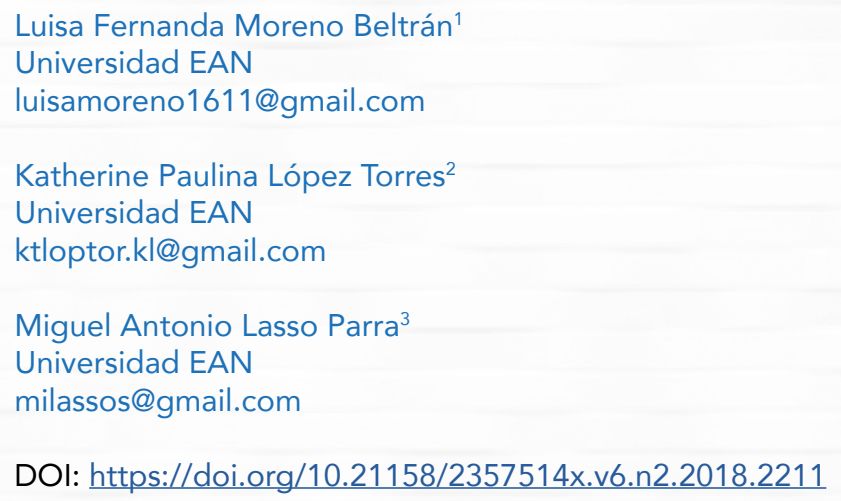




\section{RESUMEN}

El objetivo de esta investigación es evaluar el potencial y la efectividad de las palabras clave de longtail de georreferenciación, en el sector específico de la venta de bebidas naturales en Internet por medio de una campaña SEM (Search Engine Marketing). La base teórica proviene de una serie de investigaciones acerca de la clasificación de las palabras clave y el efecto que tiene la ubicación en las campañas de marketing. Para la creación de los anuncios se hizo un análisis de cómo buscaban en Internet las personas que consumen este tipo de producto, los beneficios asociados y los competidores. Se escogieron las palabras con una competencia alta y un buen nivel de búsquedas. Para medir la efectividad de estos términos de búsqueda se decidió correr una campaña de seis anuncios en Google por 10 días. Con el análisis de los resultados se identifico que la característica de geolocalización solo es usada por marcas líderes del mercado y franquicias con presencia en diferentes ciudades.

Palabras clave: marketing; palabras clave; longtail; geolocalización; anuncios de búsqueda; SEM; CPC. 


\section{Geolocation in Search Engine Marketing campaigns: a look at the use of location terms in keywords}

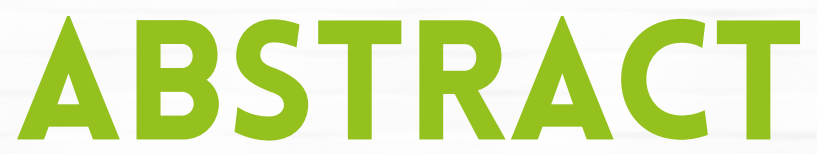

The aim of this research is to evaluate the potential and effectiveness of geo-referencing longtail keywords in the specific sector of online sales of natural beverages by means of a Search Engine Marketing (SEM) campaign. The theoretical basis comes from a series of investigations about the classification of keywords and the effect that the location has on marketing campaigns. For the creation of the ads, we compiled an analysis on how people who consume this type of product searched for it on the Internet, the associated benefits, and the competitors. The words with high competence and a good level of searches were chosen, and then, in order to measure the effectiveness of these search terms, we ran a campaign of six ads on Google for 10 days. With the analysis of the results, we identified that only leading market brands and franchises that are present in different cities use the geolocation feature.

Keywords: marketing; keywords; longtail; geolocation; search ads; Search Engine Marketing (SEM); CPC. 


\section{Géolocalisation des campagnes SEM (Search Engine Marketing):un aperçu de l'utilisation des termes de localisation des mots clefs}

\section{RÉSUME}

L'objectif de cette recherche est d'évaluer le potentiel et l'efficacité du géoréférencement de mots-clés de type longtail dans le secteur spécifique de la vente de boissons naturelles sur Internet via une campagne de marketing sur moteurs de recherche. La base théorique provient d'une série d'enquêtes sur la classification des mots-clés et l'effet de la localisation sur les campagnes marketing. Une analyse a ensuite été réalisée sur la création des publicités et la manière dont les consommateurs de ce type de produit effectuaient des recherches sur internet. Les mots ayant simultanément la meilleure visibilité et un nombre de saisies élevé sur les moteurs de recherche ont été sélectionnés et, pour en mesurer l'efficacité, une campagne de six annonces sur Google a été lancée pendant 10 jours. L'analyse les résultats montre que la fonction de géolocalisation n'est utilisée que par les grandes marques du marché et les franchises présentes dans différentes villes.

Mots clefs: marketing, mots-clés; longtail; géolocalisation; annonces de recherche; SEM; CPC. 


\section{Geolocalização nas campanhas SEM: um olhar sobre o uso dos termos de localização nas palavras-chave}

\section{RESUMO}

O objetivo desta pesquisa é avaliar o potencial e a efetividade das palavras-chave de longtail de georreferenciação, no setor específico de venda de bebidas naturais em Internet por meio de uma campanha SEM (Search Engine Marketing). A base teórica vem de uma série de pesquisas a respeito da classificação de as palavras-chave e o efeito que tem a localização nas campanhas de marketing. Para a criação dos anúncios fez-se uma análise de como procuravam em Internet as pessoas que consomem este tipo de produto, os benefícios associados e os competidores. Escolheram-se as palavras com uma concorrência alta e um bom nível de buscas. Para medir a efetividade destes termos de busca decidiu-se usar uma campanha de seis anúncios em Google por 10 dias. Com a análise dos resultados identificamos que a característica de geolocalização só é usada por marcas líderes do mercado e franquias com presença em diferentes cidades.

Palavras-chave: marketing; palavras-chave; longtail; geolocalização; anúncios de busca; SEM; CPC. 


\section{Introducción}

Los usuarios y anunciantes de campañas SEM (search engine marketing) hacen uso de palabras realmente específicas para reducir, dependiendo del caso, la lista de resultados y aparecer solo en aquellas búsquedas que pueden ser transaccionales y de valor para el anunciante. Las campañas SEM también son una oportunidad para presentar anuncios a los consumidores adaptados a sus intereses de búsqueda, lo que aumenta considerablemente la probabilidad de que hagan clic en estos anuncios (Agarwal, Hosanagar y Smith, 2011). Siendo así, el objetivo de esta investigación es evaluar la efectividad que pueden tener las palabras clave de longtail con geolocalización en los anuncios en buscadores.

Esta investigación se planteó como hipótesis qué las palabras relacionadas con la ubicación, como ciudades o barrios, pueden ser más transaccionales y rentables para pautar, ya que le quitan ambigüedad a la búsqueda y muestran un interés concreto del internauta. Dado que la probabilidad de que un consumidor haga clic en un anuncio depende de la relevancia del intento del anunciante para captar el interés de búsqueda del cliente, cualquier posible desajuste entre el interés de búsqueda del consumidor y la intención del anunciante podría reducir la eficacia de la publicidad de búsqueda (Gong, Abhishek y Li, 2018, p. 806).

Para poner a prueba la hipótesis, se ejecutó una campaña SEM para un emprendimiento bogotano de batidos desintoxicantes con seis anuncios. Este artículo puede ser útil en el momento de establecer mejores pautas a la hora de hacer campañas de search en Google y ser un aporte desde lo empírico a la construcción de conocimiento acerca de las palabras clave geolocalizadas, ya que en este momento el estado actual relacionado no es muy extenso.

\section{Revisión de literatura}

Este estudio se basa en la importancia de la optimización de campañas SEM, que constituye un factor importante a considerar en el desarrollo del trabajo de investigación, gracias a la facilidad que proporciona para ejecutar, medir e interpretar los resultados de las campañas y al hecho de que pretende incrementar la visibilidad de las páginas web en los motores de búsqueda haciéndolas más efectivas. SEM permite ir más allá, facilita la interpretación de aquello que los consumidores prefieren y aquello que posiblemente preferirían.

Como lo afirman Clarke, Murphy, Wetsch y Boeck (2018), SEM es una forma establecida de mejorar la eficacia en línea. Usar el marketing de motores de búsqueda es una forma relevante y responsable de comercialización dentro del cada vez más competitivo espacio del mercado digital (p.136). Para esta investigación, es relevante la optimización de campañas SEM, pues se parte de la máxima de que el 
uso de las herramientas que se ofrecen por defecto para la optimización, como la geolocalización, ya están previamente confrontadas.

Este factor es importante considerarlo, pues podría estar ligado a la efectividad en los buscadores, que determina cuán rápida y pertinente es la información que encuentran los usuarios después de ubicar en el cuadro de búsqueda la palabra clave determinada. Se debe asegurar que la función de búsqueda sea efectiva. A pesar de ser una búsqueda una actividad tan importante, una sorprendente cantidad de sitios web la hacen mal. No hay que ser uno de ellos (Bloomsbury Business Library, 2007).

Teniendo en cuenta que las palabras clave pueden afectar la percepción pública, la mentalidad del usuario $y$, por supuesto, las clasificaciones del anuncio, este ejercicio de investigación intenta comprender el poder del uso coherente de las palabras clave adecuadas y cómo estas construyen un lado positivo que puede ayudar a impulsar al éxito a las organizaciones.

Las palabras clave contribuyen al éxito de un anuncio en publicidad online. La efectividad de la publicidad en los buscadores se atribuye al hecho de que la búsqueda en ellos coincide con la exposición de los anuncios a un consumidor con el intento de búsqueda actual, derivado de la palabra clave que se está buscando. La capacidad de presentar anuncios a los consumidores adaptados a sus intereses de búsqueda -según lo indicado por las palabras clave- aumenta considerablemente la probabilidad de que hagan clic en estos anuncios (Gong, Abhishek y Li, 2018, p. 806).
Por otro lado, un comprador que usa palabras específicas, caso afín al de este estudio, en su consulta en general sabe exactamente lo que quiere y se encuentra en una fase posterior de su ciclo de compra. Estas palabras clave no se buscan con tanta frecuencia y no son tan populares.

Si se desarrolla una campaña en la que se utilizarán palabras clave muy específicas, no se obtendrán tantas impresiones, pero la cantidad de clics y su CTR (click through ratio) será mucho mayor. Esto se debe, principalmente, a que las personas que usan esas palabras clave de cola larga saben exactamente lo que están buscando, y si aparece un anuncio, es probable que hagan clic en su página de destino. Si lo que se está buscando son usuarios en línea que se conviertan en clientes, las palabras clave específicas deben estar en la estrategia (Jones, 2011).

Debido a estas prácticas establecidas en las campañas SEM tradicionales, se toma la efectividad del anuncio como una variable válida a contemplar en el desarrollo de esta investigación. para el caso práctico del ejercicio, se identificará el impacto de longtail, con características de ubicación, en el CTR o clic to rate que es un índice significativo para medir el efecto de la colocación de publicidad (Jiang, 2018, p. 1) consistente en el número de clics que obtiene un enlace respecto de su número de impresiones. Así, se busca explorar si una estrategia SEM basada en longtail de geolocalización puede obtener un mejor CTR que una estrategia SEM que no cuente con esta característica específica. 
Esta variable no se escogió de manera aleatoria, pues se tuvieron en cuenta los beneficios de usar palabras clave de cola larga:

- En su mayoría, pueden generar una gran cantidad de volumen de búsqueda en su sitio.

- Por lo general, son menos competitivos y, por tanto, dan como resultado un menor costo por clic (CPC) y tasas de clics (CTR) más altas.

- Dado que son más específicos, los anuncios y las páginas de destino pueden adaptarse mejor y ser más relevantes para satisfacer las necesidades de los usuarios.

- Los usuarios que utilizan palabras clave de cola larga suelen estar más avanzados en el ciclo de compra y están más cerca de tomar una decisión de compra.

- Con un CPC más bajo, un CTR más alto y tasas de conversión más altas (CVR), las palabras clave de cola larga pueden ser muy rentables (Jones, 2011).

Estas palabras clave son muy eficaces porque hay poca competencia para la mayoría de ellas en los principales buscadores. La investigación exhaustiva de palabras clave de cola larga puede ser una estrategia altamente efectiva, ya que las personas que realizan búsquedas de larga cola son posiblemente más avanzadas en el ciclo de compra - se necesita una cantidad significativa de conocimiento previo para que el buscador sepa qué palabras usar-, entonces las tasas de conversión pueden ser más altas.
Si se hace con habilidad, una campaña de búsqueda pagada con palabras clave de cola larga puede exhibir un CTR considerablemente más alto que uno con palabras clave de cola corta. Además, los precios de clic de búsqueda pagada (CPC) varían en función de una subasta de oferta y demanda en tiempo real, lo que implica que las colas largas a menudo pueden ser más baratas hasta en un $50 \%$ debido a una menor competencia (Wilson, 2012, pp. 128-129).

De igual manera, es importante para los anunciantes considerar cómo los usuarios realizan la búsqueda de sus productos o servicios online, cuáles son las palabras clave que usan en la búsqueda, puesto que el análisis de ese comportamiento permite entender sus preferencias y el camino que recorren online hasta encontrar producto final.

Para identificar los factores clave dentro de la investigación, lo primero que se hizo fue entender la efectividad del longtail desde la perspectiva teórica. Según Wilson (2012), cuando se toman en conjunto, pueden proporcionar un tráfico objetivo significativo que convierte bien y es poco probable que la cola larga exceda las búsquedas de una marca si la marca está razonablemente bien establecida (p. 128).

En consecuencia, el uso de longtail palabras clave asociadas a la ubicación se constituye en un factor importante en el momento en que el consumidor realiza una búsqueda específica; este consumidor está buscando un producto o servicio en un lugar en específico, porque le gustó más, es de mejor calidad, menor precio o estuvo más cerca a su casa. Y de acuerdo con Banerjee y Dholakia (2008), poner la información en el contexto correcto hace que parezca más útil 
como similitud entre los anuncios y los contextos publicitarios, por lo que mejora su efectividad (p. 70). Pero este punto por sí solo no ahonda en la relevancia de la geolocalización en el anuncio, por esta razón este trabajo se permite indagar el tema.

Hoy en día, el consumidor es un buscador constante de información y por tanto sabe dónde encontrar aquello que quiere y por esto al realizar una búsqueda en algunos casos escribirá la ubicación en la que este quiere encontrar lo que busca.

Las personas intentarán localizar su búsqueda con modificaciones de palabras clave que no son solo el nombre de la ciudad. Considere el condado, el estado, el código postal y los vecindarios. Haga una lista de los vecindarios, condados, códigos postales, etc., que sirva su empresa. Tenga en cuenta que su empresa puede atender múltiples regiones dependiendo de dónde se encuentre realmente. También puede servir solo una pequeña porción de una ciudad más grande. La optimización para la búsqueda local es un proceso que hace que los motores de búsqueda y los posibles visitantes sepan dónde se encuentra. Querrá optimizar su sitio web o sus blogs para ayudarlo a lograr una mayor visibilidad con los motores de búsqueda (Jones, 2011).

Como se puede observar, los estudios anteriores muestran y se enfocan en la efectividad tanto de longtail como de geolocalización, pero las toman como variables separadas, por lo que muchos son los vacíos que se encuentran a la hora de medir su efectividad combinada, por esto la medición del impacto de campañas SEM basadas en estas dos variables sirve como enfoque de esta investigación.

La efectividad se entiende, como ya se mencionó, de acuerdo con el impacto que tengan los anuncios y la respuesta que generaron, por lo que se evaluó el CTR, la posición promedio del anuncio, el número de impresiones y las conversiones que alcanzó cada anuncio, con el fin de evidenciar si el uso de palabras clave de longtail con geolocalización tienen un mejor desempeño.

\section{Pregunta de investigación e hipótesis}

Es de conocimiento general que la ubicación de un negocio es un factor crítico para el éxito, como lo plantean Zhang, Pelechrinis y Lappas (2018), las empresas en áreas urbanas densas están expuestas a más clientes que las empresas en vecindarios escasamente poblados, mientras que la proximidad a un punto de referencia popular puede aumentar el alcance de un negocio (p. 36).

Los negocios pequeños o emprendimientos que no están ubicados dentro de una zona de gran afluencia de público tienen, incluso, más difícil la tarea de atraer clientes y las empresas ajenas a este "círculo de ganadores» se enfrentan a una batalla cuesta arriba en su esfuerzo por ampliar su alcance y aumentar su cuota de mercado (Zhang et al, 2018, p. 36). 
Esto también impacta las iniciativas $100 \%$ digitales, que se deben anclar en estrategias de promoción online para alcanzar sus objetivos de mercado. Teniendo en cuenta esto, se puede afirmar que la importancia de usar la georreferenciación en digital es proporcional a la necesidad de atraer clientes a un negocio.

Por lo general, los negocios pequeños o virtuales se anclan en promociones en redes sociales que cuentan con la característica de georreferenciación y algunas en anuncios patrocinados en buscadores. Frente a esto, Zhang et al. (2018) sostienen que un negocio con un perfil en locationbased social networks (LBSN) puede ser descubierto por clientes potenciales que de otro modo podrían desconocer su presencia (p. 37). Así es como las redes sociales y la pauta digital se convierten en factores que pueden ayudar al éxito de los negocios o de las empresas.

En otras investigaciones, se ha planteado la influencia de la pauta en redes sociales con tecnología de geolocalización para impactar las conversiones y ventas en los puntos físicos, puesto que la presencia digital ha permitido que las empresas que no se encuentran en ubicaciones privilegiadas se vuelvan visibles para los clientes cercanos (Zhang et al., 2018, p. 36).

A partir de esto se plantean preguntas en torno a la geolocalización, como ¿los anuncios patrocinados en Google que contienen dentro de las palabras clave términos relacionados con ubicación como ciudad, país o barrio pueden tener un mejor rendimiento? o ¿el uso de términos de ciudad ayuda a que las búsquedas sean menos ambiguas y así los anuncios pueden ser más eficaces?
En el momento de la revisión de la literatura, se encontraron pocos estudios relacionados con el impacto de la geolocalización en anuncios patrocinados en motores de búsqueda. Sin embargo, se halló que la ubicación es una herramienta que permite en otros canales de publicidad un mejor rendimiento de las campañas, pues permite que los anuncios se muestren en zonas específicas de acuerdo con la búsqueda o necesidad del cliente. Al conocer la ubicación de un usuario, el anunciante puede ofrecer promociones personalizadas que coincidan con la proximidad de la ubicación a las empresas cercanas (Zhang et al., 2018, p. 38).

Por otro lado, Banerjee y Dholakia (2008) estudiaron la efectividad de la publicidad móvil y sus hallazgos indican que la ubicación real del cliente, así como el contexto de esa ubicación, influyen significativamente en la efectividad potencial de estrategias publicitarias.

De tal manera nos planteamos la hipótesis: una estrategia SEM basada en longtail de geolocalización para un emprendimiento de bebidas naturales en Bogotá puede obtener un mejor CTR que una estrategia SEM que no cuente con esta característica específica, de modo que es la geolocalización la variable que determina la investigación y los tipos de anuncios que se realizaron. 


\section{Método}

Se decidió seguir un diseño metodológico cuasiexperimental, puesto que los objetos de estudio no se toman de manera aleatoria —como en el modelo experimental-, sino que consisten en un conjunto preestablecido. Tal grupo de objetos constituye la variable independiente, las palabras clave de geolocalización, que es tratada con el fin de determinar su efecto o relación con las variables dependientes, que en este caso es el CTR y las bebidas naturales (Hernández, Fernández y Baptista, 2014).

Para el desarrollo de la investigación y del anuncio, se procedió a elegir seis palabras clave de tipo longtail relativas a bebidas naturales para adelgazar, que es otra de las variables dependientes. Dichas palabras se agrupan en dos categorías: general y geolocalización.

Como paso siguiente, a modo de prueba piloto se puso en marcha una campaña en la que se promueve el jugo verde para adelgazar de la empresa Vitalfrut, teniendo como objetivo medir la cantidad de clics, impresiones e impacto en el CTR de las palabras clave seleccionadas en la campaña propuesta. A continuación, se explican las fases en las que se llevó a cabo esta prueba.

\subsection{Identificación y análisis preliminares}

En este primer momento, se identifican las palabras clave relacionadas con la promesa de valor y el producto del emprendimiento. También se determina la forma en que este tipo de productos son comúnmente buscados en la web. En consecuencia, se obtiene una base de 50 palabras clave posibles (tabla 1).

Tabla 1. Términos de búsqueda

\section{Término de búsqueda}

jugo verde para bajar de peso en 7 días

jugo verde para bajar de peso

receta jugos verdes para adelgazar

jugos efectivos para bajar de peso

contenido de jugo verde para adelgazar

herbalife bogota puente aranda

cómo hacer jugo verde para bajar de peso

cómo se prepara el jugo verde para bajar de peso

jugos verdes para adelgazar

jugos verdes para bajar de peso

jugo verde para adelgazar

jugos verdes para bajar de peso recetas

los jugos verdes para bajar de peso de colan

jugos verdes para adelgazar en 5 dias

Fuente. Elaboración propia. 
A continuación, se emplea la herramienta AdWords para valorar las palabras clave encontradas de acuerdo con los siguientes parámetros: promedio de búsquedas, es decir, qué tanto son usadas en el buscador, nivel de competencia y montos de ofertas, tanto del mínimo como del máximo.

Como se tenía un presupuesto reducido, se seleccionan las palabras que tuvieran un promedio de búsquedas alto, con un nivel de competencia medio y bajo para que el valor de la puja no fuera muy elevado. Posteriormente, se escogen las tres palabras clave de longtail sin característica de ubicación para la campaña.

Como el producto que se va a promover se distribuye únicamente en Bogotá y el objetivo es descubrir si la geolocalización tiene un impacto en el CTR de la campaña, resulta relevante medir el efecto de las palabras con términos de geolocalización. Por tal motivo, se repite el procedimiento, esta vez añadiendo al final de las palabras la ubicación y eligiendo otras tres palabras para pautar.

Es importante destacar que una de las palabras clave dentro de esta categoría es Herbalife Bogotá, con el fin de determinar qué beneficio trae el uso de una marca reconocida en la categoría del producto -productos para bajar de pesoa la campaña, teniendo en cuenta su empleo en anuncios con la característica de ciudad.

\subsection{Desarrollo de la campaña}

Tras encontrar las palabras clave afines y teniendo en cuenta el presupuesto y el impacto, se empieza a ejecutar la campaña de mercadeo durante 10 días en la que se promociona el jugo verde para adelgazar de Vitalfrut. La campaña inicia con seis anuncios, tres con las palabras clave generales y tres anuncios con palabras clave de geolocalización, con el fin de poder medir el impacto y el CTR de los anuncios de la categoría general y la categoría geolocalizada.

Cada anuncio se redactó con las palabras clave seleccionadas y las cualidades del producto, también se activó la concordancia de frase, lo cual mejora la asertividad en las búsquedas que están relacionadas con las palabras clave seleccionadas y disminuye la posibilidad de recibir clics que no sean relativos al producto.

\subsection{Recolección de datos y análisis de resultados}

Para la recolección de datos y la verificación de la hipótesis, se realiza un seguimiento diario de las impresiones, CPC, clics y CTR de los seis anuncios, identificando por día cuál de las palabras o anuncios tuvo mayor cantidad de impresiones y clics. Tales datos se dividen en dos categorías: ubicación y característica general. La información obtenida es segmentada para que solo aparezcan las búsquedas realizadas en Bogotá por personas entre 18 y 44 años. De esta manera, se hace posible analizar los resultados y evaluar la hipótesis planteada. 


\section{Resultados}

Al finalizar la campaña, se encuentra que esta alcanzó 83 clics, 718 impresiones y un CTR general de la campaña del $12 \%$. La campaña tuvo picos más pronunciados por temas de horarios de búsqueda y el manejo del presupuesto manual, lo que muchas veces no permitió que los anuncios se vieran todo el tiempo (Figura 2).

Figura 2. Análisis total de clics impresiones y CTR general de la campaña

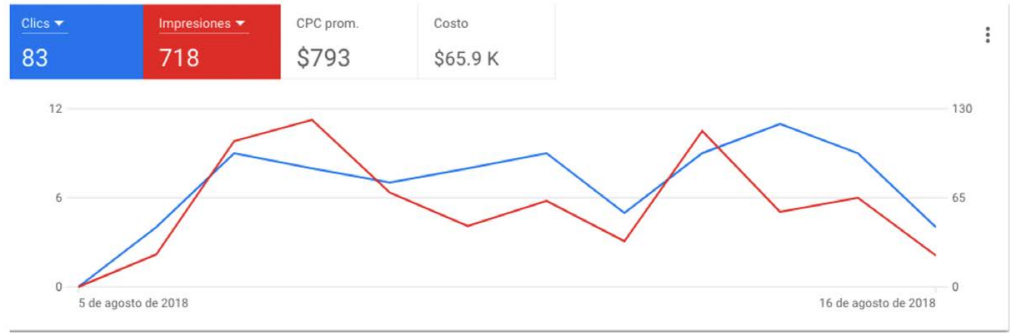

Fuente. Elaboración propia.

Las palabras clave que más clics e impresiones tuvieron fueron «jugo verde para adelgazar», «Herbalife Bogotá» y «jugo verde para bajar de peso», cada una con un CTR del 15,74 , el 5,67 y el $14,61 \%$, respectivamente (Tabla 2 ).
Tabla 2. Análisis CTR de los anuncios

\begin{tabular}{|c|c|c|c|c|c|c|c|c|c|c|}
\hline 口・ & Palabbra clave & Grppo o de anuncios & Etratado & cPe max. & clies & impr. & cTR & cecprom. & $\downarrow$ costo & 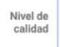 \\
\hline$\square \bullet$ & 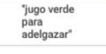 & Grupo de anuncoss 1 & 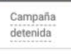 & $\operatorname{cop} 4000<1]$ & 51 & 324 & $15.74 \%$ & cope6 1 & coppas.919 & $6 / 10$ \\
\hline 口・ & 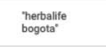 & Grppo de anuncios 1 & $\begin{array}{l}\text { campana } \\
\text { detention }\end{array}$ & coppl,19e区 & 17 & 300 & 5678 & copess & cop14,510 & 3/10 \\
\hline 口・ & 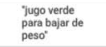 & Grupo de anuncios 1 & $\begin{array}{c}\text { camporat } \\
\text { detendica }\end{array}$ & coppr,19e日 & 13 & 99 & $14.61 \%$ & coposos & copos,568 & 6/10 \\
\hline
\end{tabular}

Fuente. Elaboración propia.

Durante los días que estuvo activa la campaña, los dos grupos de anuncios tuvieron impresiones todos los días, pero solo el grupo general tuvo una frecuencia de actividad/clics todos los días, mientras que para aquellos que contaban con georreferenciación solo 8 días el número de clics frente a las impresiones es mínimo (Tablas 3 y 4).

Tabla 3. Análisis de anuncios geolocalizados

\begin{tabular}{|c|c|c|c|c|c|c|c|}
\hline \multicolumn{8}{|c|}{ Geolocalización } \\
\hline Día & -1 Titulo & - Título 1 & Titulo 2 & Clics & .81 & In.7 CTR & - Posición pr - \\
\hline 7-ago- & & Venta de & jug Bogota & & 2 & $385.26 \%$ & 1.47 \\
\hline 7-ago-: & & Jugo ver & de p Bogota & & 2 & $825.00 \%$ & 1.25 \\
\hline 8-ago-: & & Venta de & jug Bogota & & 1 & $452.22 \%$ & 1.24 \\
\hline 9-ago-: & & Jugos de & sintc Bogota & & 1 & $250.00 \%$ & 1.00 \\
\hline 9-ago-: & & Jugo ver & de p Bogota & & 1 & $137.69 \%$ & 1.23 \\
\hline 10-ago-: & & Venta de & jug Bogota & & 1 & $128.33 \%$ & 1.42 \\
\hline 11-ago- & & Jugos de & sintc Bogota & & 2 & $450.00 \%$ & 1.25 \\
\hline 11-ago- & & Jugo ver & de $p$ Bogota & & 2 & $1711.76 \%$ & 1.47 \\
\hline 13-ago-: & & Jugo ver & de p Bogota & & 2 & $1910.53 \%$ & 1.21 \\
\hline 14-ago- & & Jugo ver & de p Bogota & & 1 & $812.50 \%$ & 1.50 \\
\hline 15-ago- & & Jugo ver & de p Bogota & & 1 & $166.25 \%$ & 1.31 \\
\hline
\end{tabular}

Fuente. Elaboración propia. 
Tabla 4. Análisis de anuncios generales

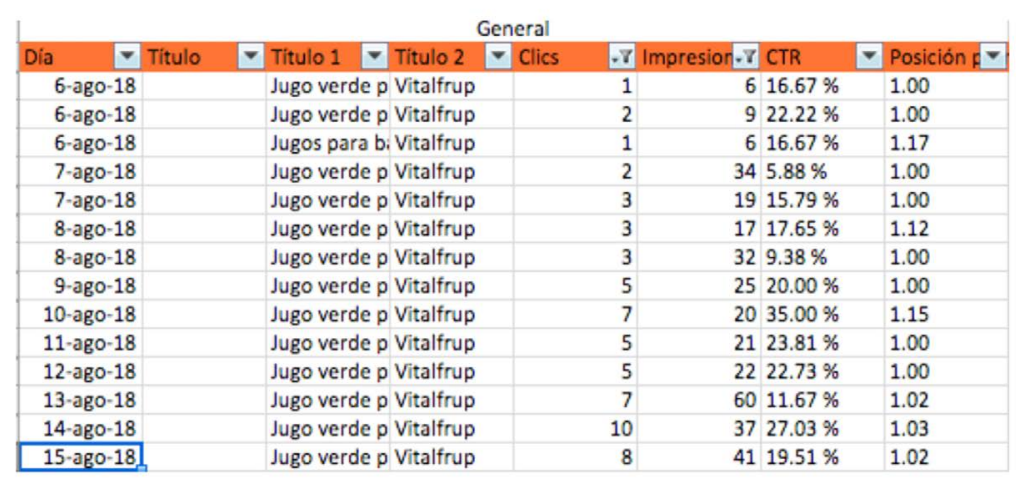

Fuente. Elaboración propia.

Durante los primeros días que estuvo activa la campaña, se pudo observar que el número de impresiones y clics era bastante bajo, pero todos los clics venían de los anuncios generales. En los primero tres días, los anuncios generales tuvieron 9 clics y 79 impresiones, mientras que los anuncios geolocalizados 4 clics y 51 impresiones (Tabla 5).

Tabla 5. Análisis de primeros días

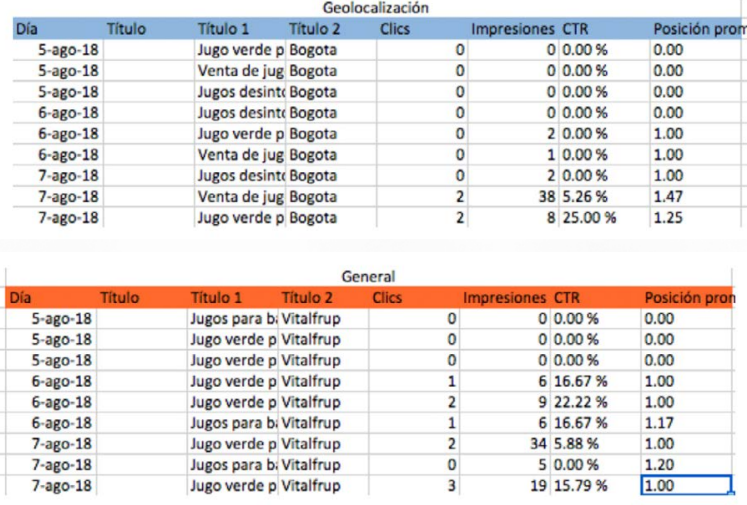

Para el resto del periodo de la campaña, el número de impresiones de ambos grupos de anuncios creció sustancialmente, hasta llegar a 718 para el último día. Sin embargo, la diferencia en clics se mantuvo. El grupo de geolocalizados nunca llegó a más de dos clics, mientras que el grupo general en su mejor día logró 10 (Tabla 6).

Tabla 6. Análisis de últimos días

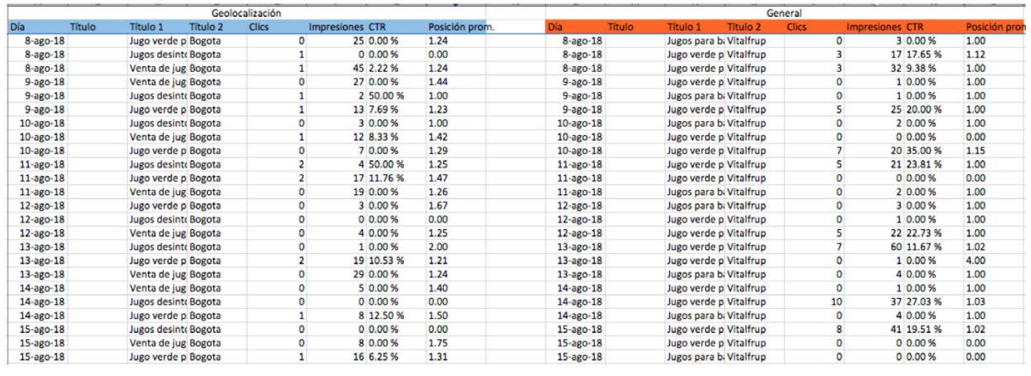

Fuente. Elaboración propia.

Evaluando por grupos de anuncios, podemos ver que la categoría general tiene un mejor rendimiento, con 64 clics, 390 impresiones y un CTR del $16 \%$, mientras que la categoría geolocalizada le aportó a la campaña 19 clics de 328 impresiones con un CTR del 6 \% (Tabla 7).

Tabla 7. Análisis de desempeño

\begin{tabular}{|c|c|c|c|}
\hline Desempeñ॰ & Clics & Impr. & CTR \\
\hline Geolozalizado & 19 & 328 & $6 \%$ \\
\hline General & 64 & 390 & $16 \%$ \\
\hline Total & 83 & 718 & $12 \%$ \\
\hline
\end{tabular}

Fuente. Elaboración propia. 
Para concluir los resultados, podemos ver que todas las métricas de clics, impresiones y CTR tuvieron un mejor desempeño en el grupo de anuncios generales en comparación con el grupo de geolocalizadas, lo que implica que la variable independiente de geolocalización no demuestra tener un impacto o mayor efectividad en relación con la variables dependientes, CTR y bebidas naturales.

De igual manera, se puede observar que el número de impresiones para el grupo geolocalizado también fue menor en comparación con los anuncios generales. Sin embargo, en lo que se refiere a posición, los dos grupos aparecieron entre el primero y segundo puesto, lo que evidencia que el CPC que se manejó era el adecuado para la puja, con lo que se permitió que se tuvieran 83 clics, de los cuales no hubo ni una solo conversión o venta, pero si se logró crecer en las cuentas de Facebook e Instagram (Figuras 3 y 4).

Figura 3. Número de clics e impresiones longtail generales versus geolocalización

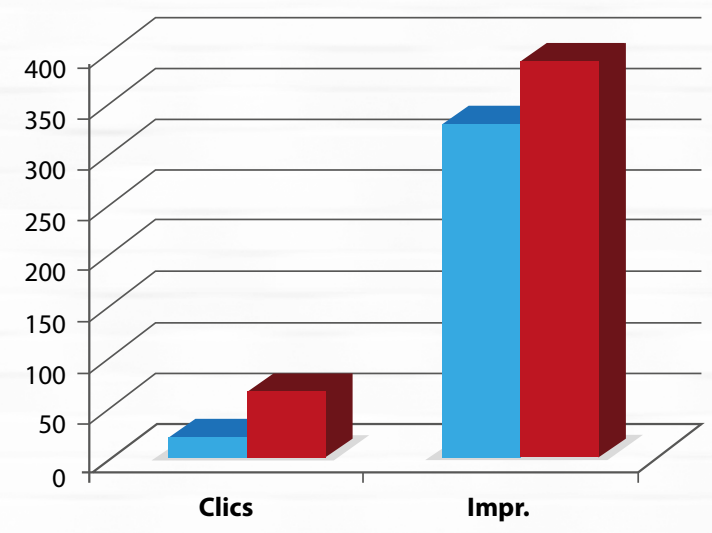

Geolocalizado

- General
Figura 4. Porcentaje CTR para longtail generales versus geolocalizadas

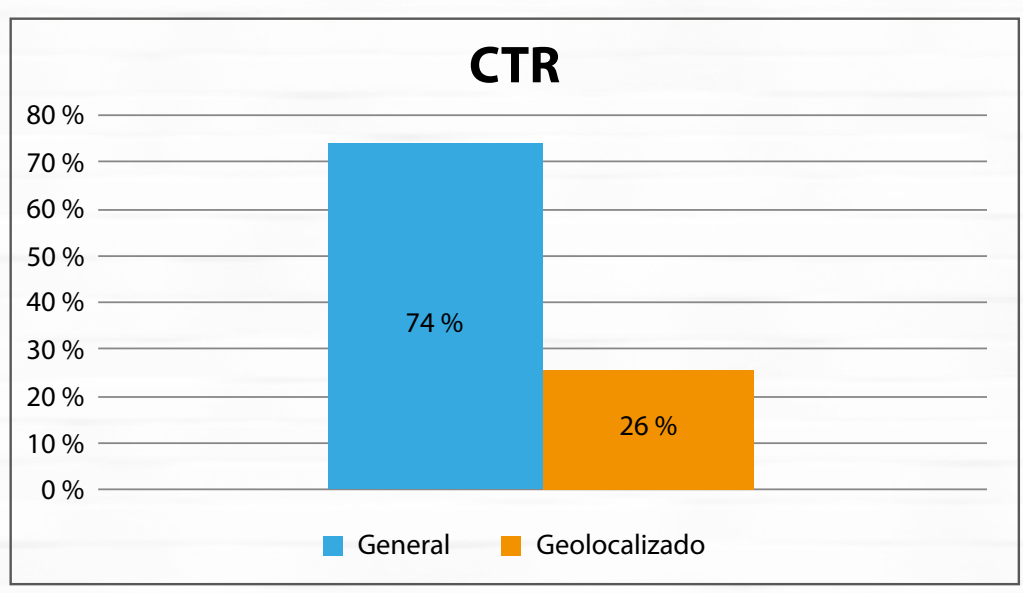

Fuente. Elaboración propia.

\section{Discusión de los resultados}

Tras analizar los resultados podemos concluir que en la categoría de bebidas naturales no se hacen muchas búsquedas con ubicación y solo las marcas líderes como Cosechas y Herbalife utilizan la geolocalización como palabra para pautar, ya que son productos posicionados que se encuentran en diferentes zonas del país y el uso de palabras de ubicación ayuda a que la búsqueda sea menos ambigua.

Es claro también en los resultados que el impacto y la performance de los anuncios de características generales fue mucho mejor que el de la categoría geolocalizada, debido a que tuvo un mayor número de clics, porque los términos que se usaron para los anuncios son los comunes para buscar este 
tipo de productos. Esto implica que en el CTR la medida de efectividad que se usó fue mejor con un $16 \%$ al finalizar la campaña.

Estos resultados no permiten que la hipótesis de la investigación sea aceptada; por el contrario, muestra una poca relación entre la variable independiente geolocalización y las variables dependientes bebidas naturales y CTR. Este resultado no implica que el uso de la variable independiente, en este caso la geolocalización, no sirva para otro tipo de productos que impliquen una relación directa entre la palabra clave de búsqueda y el CTR.

Es importante tener presente que tener un presupuesto pequeño se constituye en limitación, ya que no permitió tener la campaña activa por más tiempo, lo que limita el tamaño de la muestra y por ende los resultados. Esta limitación se presenta como una oportunidad de estudio para futuras investigaciones, que harán posible medir la eficacia real del impacto del uso de palabras clave de longtail con geolocalización, en diferentes productos o categorías, puesto que por lo naturaleza de este tipo de estrategias y estudios los resultados no se pueden generalizar.

\section{Conclusiones}

En esta investigación, es difícil concluir si las palabras clave de longtail de geolocalización tienen un mejor CTR que las otras palabras, ya que la muestra que se obtuvo es muy pequeña y la categoría de producto bajo la cual se hizo el análisis no necesariamente usa la ubicación en las búsquedas ni en los anuncios, de tal manera proponemos profundizar en este este tema desde otras categorías de producto o con marcas ya establecidas.

Los resultados obtenidos en el estudio realizado no fueron suficientes para establecer una conclusión definitiva respecto de la efectividad que pueden tener las palabras clave de longtail con geolocalización en los anuncios en buscadores, debido a algunas variables, como tipo de producto, tiempo de campaña, no asociación del usuario por producto con la palabra clave georreferenciada o la generalización de la búsqueda.

Basados en la experiencia obtenida del presente estudio, podemos concluir que las palabras clave de longtail de geolocalización no han sido objeto de suficiente investigación ni aplicación en las herramientas de optimización y posicionamiento web, pero sí constituyen un objeto de estudio sobre el cual se puede inferir algunas suposiciones e hipótesis que requieren comprobación y podrían dar lugar a un estudio y conclusiones de mayor contenido y envergadura.

Además, hace falta utilizar métodos con mayor control sobre las variables de estudio, quizá de orden experimental, que permitan dar el rigor suficiente al tema de estudio y más contundencia en cuanto a los resultados esperados, ya que el método que se usó no permite sacar una conclusión definitiva por las limitaciones que tiene. 


\section{Referencias}

Agarwal, A., Hosanagar, K. y Smith, M. D. (2011). Location, location, location: An analysis of profitability of position in online advertising markets. Journal of Marketing Research, 48(6), 1057-1073. Recuperado de https://bit.ly/2vDyEpz.

Banerjee, S. S. y Dholakia, R. R. (2008). Mobile advertising: Does location based advertising work? International Journal of Mobile Marketing, 3(2), 68-75. Recuperado de https://bit. ly/2Y4SLJK

Bloomsbury Business Library. (2007). Business \& management dictionary. Londres, RU: A y C Black Publishers.

Clarke, T. B., Murphy, J., Wetsch, L. R. y Boeck, H. (2018). Teaching search engine marketing through the google ad grants program. Marketing Education Review, 28(2), 136-147. https://doi.org/10.1080/10528008.2018.1448282

Gong, J., Abhishek, V. y Li, B. (2018). Examining the impact of keyword ambiguity on search advertising performance: A topic model approach. MIS Quarterly, 42(3), 805-829. http:// dx.doi.org/10.2139/ssrn.2404081
Hernández Sampieri, R., Fernández Collado, C. y Baptista Lucio, M. del P. (2014). Metodología de la investigación (6. ${ }^{a}$ ed.). Ciudad de México, México: McGraw-Hill.

Jiang, Z.; Gao, S. y Li M (2018) An improved advertising CTR prediction approach based on the fuzzy deep neural network. PLoS ONE 13(5). https://doi.org/10.1371/journal. pone.0190831

Jones, R. (2011). Keyword intelligence: Keyword research for search, social, and beyond. John Wiley \& Sons.

Wilson, A. (2012). Handbook of digital marketing and prominent web services. White World Publications.

Zhang, K., Pelechrinis, K. y Lappas, T. (2018). Effects of promotions on location-based social media: Evidence from foursquar. International Journal of Electronic Commerce, 22(1), 36-65. https://doi.org/10.1080/10864415.2018.1396118 